\title{
Б.В. Кавалеров, В.В. Кошелев
}

Пермский национальный исследовательский политехнический университет, Пермь, Россия

\section{МАТЕМАТИЧЕСКОЕ МОДЕЛИРОВАНИЕ ДИНАМИКИ ЭЛЕКТРИЧЕСКИХ МАШИН В ФАЗНЫХ ОСЯХ}

\begin{abstract}
В статье на примере синхронного генератора рассматривается описание динамической математической модели электрической машины. Указанное математическое описание представлено в естественной трехфазной системе координат $a, b, c$. Показаны преимущества и недостатки такого описания. На примере синхронного генератора получена общая форма записи для произвольного элемента электрической системы. Данная форма записи получена в векторноматричном виде. В такой общей форме записи целесообразно представить все основные элементы электрической системы (электрогенераторы, электродвигатели, элементы электрической нагрузки, линии электропередачи и др.) для того, чтобы можно было их совместно моделировать в рамках единой электрической системы. Показано, что при таком математическом описании индуктивности электрической машины зависят от положения ротора. Рассмотрены два варианта учета указанной зависимости: с помощью аналитических выражений и с помощью матричных преобразований. Современная компьютерная техника не встречает каких-либо сложностей при математическом моделировании электрических машин непосредственно в естественной трехфазной системе координат. Широкое использование преобразованной двухфазной системы координат во многом может быть объяснено традицией. Описание электрических машин в осях $a, b, c$ выглядит особенно целесообразным при исследовании несимметричных режимов работы в электрических системах и несимметричной конструкции электрической машины. Математические модели, рассмотренные в статье, могут использоваться для изучения динамических процессов в электрических системах, в том числе несимметричных динамических процессов. На базе полученного в статье математического описания планируется создать оригинальный программный комплекс для моделирования разнообразных динамических режимов трехфазных электрических систем.
\end{abstract}

Ключевые слова: электрическая система, электрическая машина, синхронный генератор, математическое моделирование. 


\section{B.V. Kavalerov, V.V. Koshelev}

Perm National Research Polytechnic University, Perm, Russian Federation

\section{MATHEMATICAL MODELLING OF DYNAMICS OF ELECTRICAL MACHINES IN-PHASE AXES}

In the article on the example of synchronous generator the description of dynamic mathematical model of electric machine is considered. This mathematical description is presented in the natural threephase coordinate system a,b,c. The advantages and disadvantages of this description are shown. On the example of a synchronous generator, a General form of recording for an arbitrary element of the electrical system is obtained. This form of recording is obtained in vector-matrix form. In such a General form of recording it is advisable to present all the main elements of the electrical system (generators, electric motors, electric load elements, power lines, etc.) in order to be able to model them together within a single electrical system. It is shown that in this mathematical description of the inductance of the electric machine depends on the position of the rotor. Two variants of the account of the specified dependence are considered: by means of analytical expressions and by means of matrix transformations. Modern computer technology does not encounter any difficulties in mathematical modeling of electric machines directly in the natural three-phase coordinate system. The widespread use of the transformed two-phase coordinate system can be largely explained by tradition. Description of electrical machines in the axes a,b,c seems particularly appropriate in the study of asymmetric modes of operation in electrical systems and the asymmetric design of the electric machine. This article is the first article. Following it in the next article will be considered modeling of interaction of electric machines and other elements among themselves within the framework of a single electrical system. Mathematical models considered in the article can be used to study dynamic processes in electrical systems. Including asymmetric dynamic processes. On the basis of the mathematical description obtained in the article, it is planned to create an original software package for modeling various dynamic modes of three-phase electrical systems.

Keywords: electrical system, electrical machine, synchronous generator, mathematical modeling.

Введение. При исследовании поведения электрических систем (ЭС) очень большую роль играет математическое моделирование электромеханических и электромагнитных динамических процессов, протекающих в таких системах. Эти процессы очень разнообразны, и их характер зависит от множества факторов, например, от состава элементов электрической системы, конфигурации этой системы, рассматриваемой режимной ситуации. Математическое моделирование приходит на помощь и в тех случаях, когда провести эксперименты на реальном объекте затруднительно, например, при исследовании разнообразных аварийных режимов ЭС. В этом случае благодаря моделированию сам эксперимент становится компьютерным, выполняется на ЭВМ, что значительно сокращает труд исследователя. Но математическое описание и вслед за этим компьютерное моделирование многоэлементных ЭС разнообразной конфигурации являются также непростыми задачами. Безусловно, исследователь может воспользоваться 
одним из имеющихся на рынке пакетов математического моделирования. Современные средства визуального моделирования, как правило, поддерживают язык функциональных блоков, из которых экспериментатор составляет разнообразные схемы ЭС, в качестве широко известного примера можно привести специализированную библиотеку системы динамического моделирования Simulink (язык компьютерных вычислений MatLab). Однако для того, чтобы проводить полноценные научные исследования, необходимо иметь четкое представление о том, каким образом выполнено математическое описание того или иного элемента ЭС, как организовано их взаимодействие между собой, не говоря уже о принятых при этом допущениях. Поэтому в настоящей статье ставится и решается задача получения такого математического описания элементов ЭС, которое, во-первых, даст возможность объединить эти элементы в единую систему для совместного моделирования, а, во-вторых, будет основано на четком отображении реальной физики процессов, протекающих в реальной трехфазной электрической системе с учетом несимметрии отдельных фаз. Математическое описание рассматривается как основа для создания оригинальных программных комплексов поддержки профильных научных исследований. Материалы статьи используют ранее полученные результаты, представленные в [1, 2].

Моделирование электрических машин. Очевидно, что в ЭС наиболее сложным структурным элементом являются вращающиеся электрические машины по причине вращения их роторов и взаимного перемещения их обмоток. Кроме этого все основные элементы ЭС представляются в сложной многокоординатной (комплексной) форме записи. Это вызвано тем, что электрические переменные - токи и напряжения - являются векторами, обладающими модулем и фазой, а следовательно, одного числа для их описания недостаточно. Комплексные координаты традиционно используются при описании процессов в теоретических основах электротехники (широко известный символический метод [3]). Однако в теории электрических машин представление электрических переменных в комплексной форме определяется выбором осей отсчета. Выбор тех или иных осей отсчета определяется удобством исследователя. В.А. Веников [4] называет «оси отсчета» более удачным термином, чем «оси координат». 
Напомним наиболее распространенные оси отсчета для моделирования вращающихся электрических машин:

a) неподвижные трехфазные оси отсчета $a, b, c$, их иногда называют «естественными», поскольку они непосредственно соотносятся с реальными фазами электрической машины. Поэтому эти оси дают возможность представить переменные электрических машин в «естественном» трехфазном виде. Однако уравнения получаются громоздкими [4] из-за того, что для представления вектора на плоскости достаточно только двух координат. Три координаты не нужны. Поэтому в теории электрических машин широко применяются преобразованные двухфазные оси, которые моделируют реальные трехфазные электрические машины;

б) неподвижные оси $\alpha, \beta$, при этом напряжения и токи имеют одинаковую частоту, равную частоте тока статора. Этот подход неудобен при рассмотрении процессов в синхронных генераторах, которые управляются со стороны ротора (обмотка возбуждения расположена на роторе);

в) оси $d, q$, зафиксированные на роторе электрической машины, наиболее распространены при исследовании процессов в системах, содержащих синхронные генераторы. В этой системе осей отсчета записываются широко известные уравнения Парка-Горева, которые в настоящее время общеприняты для моделирования синхронных машин;

г) оси $x, y$, вращающиеся с синхронной скоростью магнитного поля машины. Эти оси используются в основном для моделирования асинхронных двигателей. Объясняется это тем, что асинхронный двигатель получает питание от обмоток статора синхронного генератора, поэтому синхронный генератор и определяет синхронную скорость магнитного поля машины.

Как видим, разные электрические машины традиционно моделируют в разных осях отсчета. Поэтому в ЭС, где одновременно работают и асинхронные двигатели, и синхронные машины, приходится делать выбор в пользу какой-то одной из этих систем осей $(d, q$ или $x, y)$. Ввиду того, что синхронный генератор является питающим, задающим элементом ЭС $[5,6]$, предпочтение обычно отдается осям $d, q$ $[7,4,8]$. Этот подход является традиционным. В то же время очевидная универсальность естественных фазных осей отсчета $a, b, c$ заставила нас ранее рассмотреть возможность описания элементов в этих осях $[1,2]$ применительно к моделированию структурно-сложных ЭС. 
В данной статье обсуждаются возможности и ограничения применения осей $a, b, c$ и излагаются результаты, полученные ранее $[1,2]$. Тем более, что в последнее время ряд авторов вновь обращается к трехфазным осям при описании электрических машин $[9,10]$.

Математическое описание электрической машины в фазных осях. Преимущества описания синхронных генераторов в осях $d, q$ хорошо известны, это и сокращение вычислительных затрат, вычислительных ошибок на решение дифференциальных уравнений с переменными коэффициентами, и возможности лучшего понимания физики процессов за счет рассмотрения их со стороны вращающегося ротора. Но современные возможности вычислительной техники резко снижают значимость первой причины, а понимание физики процессов может только выиграть от альтернативного рассмотрения одного и того же явления с точки зрения разных систем отсчета.

Так, уже в работе 1961 г. [11] предлагается использовать переменные коэффициенты и трехфазные оси отсчета, при этом подчеркивается, что уравнения Парка-Горева (т.е. уравнения в осях $d, q$ ) могут успешно применяться только в тех режимах, при которых не нарушается симметрия фаз электрогенератора. Вот только неполный перечень таких режимов: исследование переходных процессов электрических машин с нарушенной электрической симметрией; исследование переходных процессов при продольной и/или поперечной несимметрии сети; исследование динамических режимов электрических машин с полупроводниковыми возбудителями; различные аварийные режимы. Последнее обстоятельство объясняется тем, что возникающие несимметричные режимы работы ЭС являются, как правило, анормальными или аварийными, например, двухфазные и однофазные короткие замыкания. При этом при каждом случае несимметрии появляются свои выражения для коэффициентов уравнений Парка-Горева $(d, q$ оси), из-за чего теряется главное преимущество осей $d, q-$ их общность.

Но, с другой стороны, разработка алгоритмов моделирования электрических машин в фазных координатах имеет хорошо известные сложности [12]: периодический характер коэффициентов дифференциальных уравнений и связанная с этим необходимость уменьшения шага численного интегрирования; большее, чем в преобразованной форме, число уравнений; устоявшиеся методы определения параметров электрических машин именно в $d, q$ представлении; связанные с этим 
очевидные трудности алгоритмизации. Однако современная компьютерная техника снимает первые две сложности. При этом остальные сложности могут быть преодолены. Поэтому можно сказать, что в настоящее время повсеместное использование духфазных осей объясняется главным образом традицией, в том числе не в последнюю очередь традицией преподавания по существующим учебникам.

Рассмотрим моделирование в осях $a, b, c$ на примере синхронного электрогенератора (СГ). Уравнения в фазных осях и в абсолютных единицах для СГ могут быть записаны следующим образом:

$$
\left\{\begin{array}{l}
\mathrm{d} \Psi_{a} / \mathrm{d} t=-U_{a}-r_{a} I_{a} \\
\mathrm{~d} \Psi_{b} / \mathrm{d} t=-U_{b}-r_{b} I_{b} ; \\
\mathrm{d} \Psi_{c} / \mathrm{d} t=-U_{c}-r_{c} I_{c} ; \\
\mathrm{d} \Psi_{f} / \mathrm{d} t=U_{f}-r_{f} I_{f} ; \\
\mathrm{d} \Psi_{D} / \mathrm{d} t=-r_{D} I_{D} ; \\
\mathrm{d} \Psi_{Q} / \mathrm{d} t=-r_{Q} I_{Q} ; \\
\mathrm{d} \gamma / \mathrm{d} t=\omega ; \\
\mathrm{d} \delta / \mathrm{d} t=\omega-\omega_{0} ; \\
\mathrm{d} \omega / \mathrm{d} t=\frac{1}{T_{M}}\left(M_{T}-M_{Э M}\right) .
\end{array}\right.
$$

Выражения для расчета потокосцеплений:

$$
\left\{\begin{array}{l}
\Psi_{a}=L_{a} I_{a}+M_{a b} I_{b}+M_{a c} I_{c}+M_{a f} I_{f}+M_{a D} I_{D}+M_{a Q} I_{Q}, \\
\Psi_{b}=M_{b a} I_{a}+L_{b} I_{b}+M_{b c} I_{c}+M_{b f} I_{f}+M_{b D} I_{D}+M_{b Q} I_{Q}, \\
\Psi_{c}=M_{c a} I_{c}+M_{c b} I_{b}+L_{c} I_{c}+M_{c f} I_{f}+M_{c D} I_{D}+M_{c Q} I_{Q}, \\
\Psi_{f}=M_{f a} I_{a}+M_{f b} I_{b}+M_{f c} I_{c}+L_{f} I_{f}+M_{f D} I_{D}, \\
\Psi_{D}=M_{D a} I_{a}+M_{D b} I_{b}+M_{D c} I_{c}+M_{D f} I_{f}+L_{D} I_{D}, \\
\Psi_{Q}=M_{Q a} I_{a}+M_{Q b} I_{b}+M_{Q c} I_{c}+L_{Q} I_{Q},
\end{array}\right.
$$

где $\Psi_{a}, \Psi_{b}, \Psi_{c}$ - потокосцепления статора по $a, b, c$ осям, $\Psi_{D}, \Psi_{Q}$ - потокосцепления демпферных контуров по продольной и поперечной осям, $I_{a}, I_{b}, I_{c}$ - токи статора по $a, b, c$ осям, $I_{D}, I_{Q}$ - токи демпферных контуров по продольной и поперечной осям, $\omega_{0}$ - угловая частота вращения поля статора, $\omega$ - угловая частота вращения ротора, $\delta$ - внутренний угол машины (угол нагрузки), $\gamma$ - угол поворота ротора, $M, L-$ 
взаимоиндуктиности и индуктивности соответствующих обмоток, $M_{T}-$ момент турбины, $M_{\ni м}$ - электромагнитный момент СГ. Эквивалентирование ротора по каждой оси одним демпферным контуром оказывается вполне достаточным для анализа электромеханических процессов при скольжениях 0-0,1. При более точном моделировании приходится использовать несколько контуров (три-пять контуров по каждой оси), их параметры определяются частотными методами [13]. Следует объяснить, почему для моделирования демпферных контуров используются две оси: продольная и поперечная. Это вызвано тем, что это именно эквивалентные контуры, т.е. их на самом деле в генераторе нет, но эти два контура моделируют реальную ситуацию с демпфированием в машине. Например, в турбогенераторе, как правило, вообще нет какихлибо демпферных обмоток. Там роль демпферных токов выполняют вихревые токи в массиве ротора. Такое представление демпферных свойств синхронного генератора довольно широко применяется, например, в [14, 15].

Примем допущение о полной симметрии статора и синусоидальности наводимых ЭДС холостого хода в статоре.

При рассмотрении СГ в трехфазных координатах параметры взаимоиндукции и самоиндукции становятся периодическими функциями угла поворота ротора $\gamma$. Эти функции представлены во многих книгах [16]. Для того, чтобы получить описание модели в векторноматричном виде, рассмотрим, как меняются эти периодические параметры [16]. В случае полной симметрии статора СГ индуктивность обмотки возбуждения $L_{f}$ и индуктивности обеих демпферных обмоток $L_{D}$ и $L_{Q}$ в системе (2) постоянны (при неучете насыщения). Так как обмотка возбуждения и продольная демпферная обмотка неподвижны друг относительно друга, то неизменна взаимная индуктивность $M_{f D}$. Взаимные индуктивности между поперечной демпферной обмоткой и обмотками, расположенными по продольной оси ротора, отсутствуют: $M_{f D}=M_{D Q}=0$. Все остальные индуктивности машины в общем случае зависят от положения ротора, т.е. от угла $\gamma$. Поскольку наводимые в статоре ЭДС считаются синусоидальными, и это практически достигается в современных синхронных машинах, то закон изменения взаимных индуктивностей между обмоткой возбуждения и каждой фазной обмоткой статора (например, для фазы $a$ ) примет следующий вид:

$$
M_{f a}=M_{a f}=M_{d} \cos \gamma .
$$


Далее считаем, что при совпадении магнитной оси фазной обмотки с осью $d$ ротора приведенные к статору взаимные индуктивности между двумя любыми обмотками, расположенными по этой оси, одинаковы и составляют $M_{d}$. Например, для взаимной индуктивности между обмоткой фазы $a$ и продольной демпферной обмоткой

$$
M_{D a}=M_{a D}=M_{d} \cos \gamma .
$$

Для поперечной демпферной обмотки и обмотки фазы $a$

$$
M_{Q a}=M_{a Q}=M_{q} \sin \gamma,
$$

где $M_{q}$ - взаимная индуктивность при совпадении осей поперечных обмоток. Для фаз $b, c$ в формулы (3)-(5) следует вместо $\gamma$ записать $\gamma-\frac{2 \pi}{3}$ или $\gamma-\frac{4 \pi}{3}$ соответственно.

Теперь рассмотрим фазные обмотки СГ. Их изменения происходят из-за явнополюсности ротора при его вращении гармонически с периодом $\pi$. Поэтому для фазы $a$ имеем:

$$
L_{a}=l_{0}+l_{2} \cos 2 \gamma,
$$

и для взаимной индуктивности между обмотками фаз $a$ и $b$ :

$$
M_{a b}=m_{0}+m_{2} \cos \left(\gamma-\frac{\pi}{3}\right),
$$

где $l_{2}$ и $m_{2}$ - амплитуды вторых гармоник.

Для синхронных машин в настоящее время установилось представление в паспортах параметров через индуктивные сопротивления по осям $d$ и $q\left(x_{d}, x_{q}\right)$. Поэтому необходимы выражения, чтобы рассчитать параметры СГ в осях $a, b, c$ через параметры той же машины, но в осях $d, q$. Вывод этих соотношений содержится в [16].

В установившемся режиме при протекании по статору токов нулевой последовательности $I_{a}=I_{b}=I_{c}=I_{m}$ потокосцепление фазы с учетом (6) и (7) будет следующим:

$$
\Psi_{a}=\left\{\left(l_{0}+2 m_{0}\right)+\left(l_{2}-m_{2}\right) \cos 2 \gamma\right\} I_{m} \cos \omega t,
$$

поэтому индуктивность нулевой последовательности будет определяться как

$$
L_{0}=l_{0}+2 m_{0}+\left(l_{2}-m_{2}\right) \cos 2 \gamma
$$


Из (9) следует, что $L_{0}$ изменяется с двойной частотой относительно своего среднего значения $\left(l_{0}+2 m_{0}\right)$. Но эти изменения очень малы [16] и ими пренебрегают:

$$
l_{2}=m_{2} \text {. }
$$

В результате выражение (9) упрощается:

$$
L_{0}=l_{0}+2 m_{0} .
$$

Равенство (11) справедливо, если индукция в воздушном зазоре распределена строго синусоидально (принято как допущение). В этом случае вращение ротора не влияет на $L_{0}$.

Точно так же находится потокосцепление фазной обмотки при протекании установившихся токов прямой последовательности: $I_{a}=I_{m} \cos \gamma, \quad I_{b}=I_{m} \cos \left(\gamma-\frac{2 \pi}{3}\right), \quad I_{c}=I_{m} \cos \left(\gamma-\frac{4 \pi}{3}\right)$. Потокосцепление и индуктивность будут равны:

$$
\begin{aligned}
& \Psi_{a}=\left(l_{0}-m_{0}+\frac{3}{2} l_{2}\right) I_{m} \cos \gamma, \\
& L_{d}=\frac{\Psi_{a}}{I_{m} \cos \gamma}=l_{0}-m_{0}+\frac{3}{2} l_{2} .
\end{aligned}
$$

При токах: $I_{a}=I_{m} \sin \gamma, \quad I_{b}=I_{m} \sin \left(\gamma-\frac{2 \pi}{3}\right), \quad I_{c}=I_{m} \sin \left(\gamma+\frac{2 \pi}{3}\right)$ поперечная синхронная индуктивность

$$
L_{q}=\frac{\Psi_{A}}{I_{m} \cos \gamma}=l_{0}-m_{0}+\frac{3}{2} l_{2} .
$$

Из совместного решения (11), (13), (14) находим:

$$
l_{0}=\frac{1}{3}\left(L_{d}+L_{q}+L_{0}\right) ; l_{2}=m_{2}=\frac{1}{3}\left(L_{d}-L_{q}\right) ; m_{0}=\frac{1}{3}\left(L_{0}-\frac{L_{d}+L_{q}}{2}\right) .
$$

В результате по этим выражениям можно записать переменные индуктивности СГ по ее паспортным данным, выраженным в $d, q$ осях. Тем самым снимается важное ограничение на моделирование СГ в трехфазных осях. Решение дифференциальных уравнений (1) требует обращения матрицы индуктивностей на каждом шаге расчета, но и сами эти индуктивности периодически изменяются. 
Теперь на примере СГ получим обобщенную форму записи для различных элементов ЭС в осях $a, b, c$ за счет представления уравнений (1) и (2) в векторно-матричной форме записи. Такое представление даст возможность объединять эти модели элементов в единую сложную модель для совместного исполнения $[17,18,6,2]$. Для этого запишем уравнения синхронной машины относительно производных статорных токов:

$$
\begin{gathered}
\left(\begin{array}{ccc}
L_{a} & M_{a b} & M_{a c} \\
M_{b c} & L_{b} & M_{b c} \\
M_{c a} & M_{c b} & L_{c}
\end{array}\right) p\left(\begin{array}{l}
I_{a} \\
I_{b} \\
I_{c}
\end{array}\right)=-\left(\begin{array}{ccc}
r_{a} & 0 & 0 \\
0 & r_{b} & 0 \\
0 & 0 & r_{c}
\end{array}\right)\left(\begin{array}{l}
I_{a} \\
I_{b} \\
I_{c}
\end{array}\right)-\left(\begin{array}{l}
U_{a} \\
U_{b} \\
U_{c}
\end{array}\right)- \\
-\left(\begin{array}{ccc}
M_{a f} & M_{a D} & M_{a Q} \\
M_{b f} & M_{b D} & M_{b Q} \\
M_{c f} & M_{c D} & M_{c Q}
\end{array}\right) p\left(\begin{array}{l}
I_{f} \\
I_{D} \\
I_{Q}
\end{array}\right)-p\left(\begin{array}{ccc}
L_{a} & M_{a b} & M_{a c} \\
M_{b a} & L_{b} & M_{b c} \\
M_{c a} & M_{c b} & L_{c}
\end{array}\right)\left(\begin{array}{l}
I_{a} \\
I_{b} \\
I_{c}
\end{array}\right)- \\
-p\left(\begin{array}{lll}
M_{a f} & M_{a D} & M_{a Q} \\
M_{b f} & M_{b D} & M_{b Q} \\
M_{c f} & M_{c D} & M_{c Q}
\end{array}\right)\left(\begin{array}{c}
I_{f} \\
I_{D} \\
I_{Q}
\end{array}\right) .
\end{gathered}
$$

Запишем также уравнения синхронной машины относительно производных роторных токов:

$$
\begin{aligned}
&\left(\begin{array}{ccc}
L_{f} & M_{f D} & 0 \\
M_{D f} & L_{D} & 0 \\
0 & 0 & l_{Q}
\end{array}\right) p\left(\begin{array}{l}
I_{f} \\
I_{D} \\
I_{Q}
\end{array}\right)=\left(\begin{array}{c}
U_{f}-I_{f} r_{f} \\
-I_{D} r_{D} \\
-I_{Q} r_{Q}
\end{array}\right)-p\left(\begin{array}{lll}
M_{f a} & M_{f b} & M_{f c} \\
M_{D a} & M_{D b} & M_{D c} \\
M_{Q a} & M_{Q b} & M_{Q c}
\end{array}\right)\left(\begin{array}{c}
I_{a} \\
I_{b} \\
I_{c}
\end{array}\right)- \\
&-\left(\begin{array}{ccc}
M_{f a} & M_{f b} & M_{f c} \\
M_{D a} & M_{D b} & M_{D c} \\
M_{Q a} & M_{Q b} & M_{Q c}
\end{array}\right) p\left(\begin{array}{c}
I_{a} \\
I_{b} \\
I_{c}
\end{array}\right) .
\end{aligned}
$$

Составим новую систему на основе (15) и (16) в следующем виде:

$$
\left(\begin{array}{ll}
\mathbf{X}_{s} & \mathbf{X}_{s r} \\
\mathbf{X}_{r s} & \mathbf{X}_{r}
\end{array}\right)\left(\begin{array}{c}
p \mathbf{I}_{s} \\
p \mathbf{I}_{r}
\end{array}\right)=-\left(\begin{array}{cc}
\mathbf{r}_{s} & 0 \\
0 & \mathbf{r}_{r}
\end{array}\right)\left(\begin{array}{l}
\mathbf{I}_{s} \\
\mathbf{I}_{r}
\end{array}\right)-\left(\begin{array}{l}
\mathbf{U}_{s} \\
\mathbf{U}_{r}
\end{array}\right)-\left(\begin{array}{cc}
p \mathbf{X}_{s} & p \mathbf{X}_{s r} \\
p \mathbf{X}_{r s} & \mathbf{0}
\end{array}\right)\left(\begin{array}{l}
\mathbf{I}_{s} \\
\mathbf{I}_{r}
\end{array}\right),
$$

где индексами $s, r$ обозначены блоки матриц, размером $3 \times 3$, составленные из роторных и статорных параметров. Так как блочная матрица $\mathbf{X}_{r}$ состоит из постоянных элементов, то ее производная - нулевая матрица. Преобразуем (17): 


$$
\left(\begin{array}{c}
p \mathbf{I}_{s} \\
p \mathbf{I}_{r}
\end{array}\right)=\left(\begin{array}{ll}
\mathbf{X}_{s} & \mathbf{X}_{s r} \\
\mathbf{X}_{r s} & \mathbf{X}_{r}
\end{array}\right)^{-1}\left[-\left(\begin{array}{cc}
\mathbf{r}_{s}+p \mathbf{X}_{s} & p \mathbf{X}_{s r} \\
p \mathbf{X}_{r s} & \mathbf{r}_{r}
\end{array}\right)\left(\begin{array}{l}
\mathbf{I}_{s} \\
\mathbf{I}_{r}
\end{array}\right)\right]-\left(\begin{array}{cc}
\mathbf{X}_{s} & \mathbf{X}_{s r} \\
\mathbf{X}_{r s} & \mathbf{X}_{r}
\end{array}\right)^{-1}\left(\begin{array}{c}
\mathbf{U}_{s} \\
\mathbf{U}_{r}
\end{array}\right)
$$

Обозначим клетки обратных матриц символом (*) и получим:

$$
\left(\begin{array}{c}
p \boldsymbol{I}_{s} \\
p \boldsymbol{I}_{r}
\end{array}\right)=-\left(\begin{array}{cc}
\boldsymbol{X}_{s}^{*}\left(\boldsymbol{r}_{s}+p \boldsymbol{X}_{s}\right) & \boldsymbol{X}_{s r}^{*} p \boldsymbol{X}_{r s} \\
\boldsymbol{X}_{r s}^{*}\left(\boldsymbol{r}_{s}+p \boldsymbol{X}_{s}\right) & \boldsymbol{X}_{r}^{*} \boldsymbol{r}_{r}
\end{array}\right)\left(\begin{array}{c}
\boldsymbol{I}_{s} \\
\boldsymbol{I}_{r}
\end{array}\right)-\left(\begin{array}{c}
\boldsymbol{X}_{s}^{*} \boldsymbol{U}_{s}+\boldsymbol{X}_{s r}^{*} \boldsymbol{U}_{r} \\
\boldsymbol{X}_{r s}^{*} \boldsymbol{U}_{s}+\boldsymbol{X}_{r}^{*} \boldsymbol{U}_{r}
\end{array}\right),
$$

затем сформируем вот такое уравнение относительно производных статорных токов:

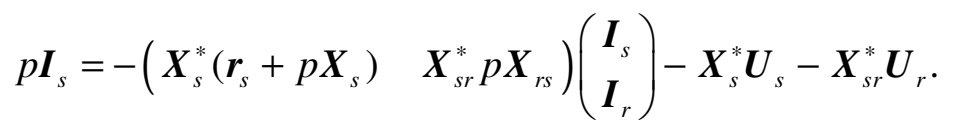

Или, введя новую обобщенную форму записи [15],

$$
p \boldsymbol{I}_{S}=-\boldsymbol{A} \boldsymbol{U}-\boldsymbol{B I}-\boldsymbol{H},
$$

где матрицы имеют следующий размер: матрица $\mathbf{A}-$ размер $3 \times 3$, матрица В - размер $3 \times 6$, вектор Н размер $3 \times 1$, вектор $\mathbf{I}-$ размер $6 \times 1$. Матрицы А и В содержат производные изменения параметров во времени.

В такой единой форме (21) были получены описания всех основных структурных элементов ЭС, не только электрических машин, но и линий связи, трансформаторов, активно-индуктивной нагрузки и др. [2]. Теперь эти элементы возможно легко соединять между собой для совместного моделирования, подобно тому, как это делается при представлении моделей элементов в осях $d, q[18,19]$. Методику объединения моделей отдельных элементов в модель ЭС, ранее описанную в [2], планируется подробно рассмотреть в следующей статье.

Для удобной алгоритмизации вместо аналитических расчетов блочных матриц в выражении (19) возможно воспользоваться другим удобным приемом $[20,12]$ на основе следующего преобразования:

$$
\mathbf{X}_{a b c}^{-1}=\boldsymbol{\Pi}^{-1} \mathbf{X}_{d q}^{-1} \boldsymbol{\Pi} .
$$

Для этого заранее получают выражения для матриц преобразования координат $\boldsymbol{\Pi}, \boldsymbol{\Pi}^{-1}$, которые позволят избавиться от необходимости обращать матрицы индуктивных сопротивлений на каждом шаге расчета. Для этого, представляя матрицу $\mathbf{X}_{d q}$ в следующем виде: 


$$
\mathbf{X}_{d q}=\left(\begin{array}{cccccc}
x_{d} & 0 & 0 & x_{a d} & x_{a d} & 0 \\
0 & x_{q} & 0 & 0 & 0 & x_{a q} \\
0 & 0 & x_{0} & 0 & 0 & 0 \\
x_{a d} & 0 & 0 & x_{f} & x_{a d} & 0 \\
x_{a d} & 0 & 0 & x_{a d} & x_{D} & 0 \\
0 & x_{a q} & 0 & 0 & 0 & x_{Q}
\end{array}\right),
$$

в соответствии с её структурой формируют матрицу сопряжения координат П в следующем виде:

$$
\boldsymbol{\Pi}=\left(\begin{array}{ll}
\boldsymbol{\Gamma} & 0 \\
0 & \mathbf{1}
\end{array}\right),
$$

где $\mathbf{1}$ - единичная матрица, $\Gamma$ - матрица преобразования координат:

$$
\Gamma=\frac{2}{3}\left(\begin{array}{ccc}
\cos \gamma & \cos \left(\gamma-\frac{2 \pi}{3}\right) & \cos \left(\gamma-\frac{4 \pi}{3}\right) \\
\sin \gamma & \sin \left(\gamma-\frac{2 \pi}{3}\right) & \sin \left(\gamma-\frac{4 \pi}{3}\right) \\
\frac{1}{2} & \frac{1}{2} & \frac{1}{2}
\end{array}\right) .
$$

Поскольку матрица П - квазидиагональная, то

$$
\boldsymbol{\Pi}^{-1}=\left(\begin{array}{cc}
\boldsymbol{\Gamma}^{-1} & 0 \\
0 & \mathbf{1}
\end{array}\right) .
$$

Результаты моделирования. На основе представленных соотношений созданы компьютерные программы для моделирования элементов электрических систем в естественной системе фазных осей $a, b, c$. Результаты моделирования показали полное совпадение с результатами моделирования в осях $d, q$. Вместе с тем моделирование в трехфазных осях подтвердило и все предполагаемые преимущества такого представления моделей, например, при исследовании несимметричных режимов работы.

Рассмотрим моделирование в трехфазных осях удаленных несимметричных коротких замыканий, которые часто имеют место на практике. 




Рис. 1. Процесс однофазного короткого замыкания фазы $a$

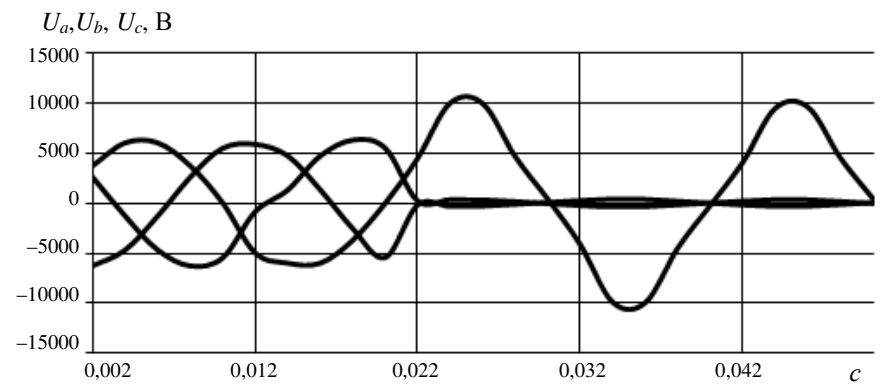

Рис. 2. Процесс короткого замыкания между фазами $b$ и $c$

Полученные результаты качественно полностью согласуются с данными, представленными в [20] и [12]. На рис. 1 и 2 представлены графики переходных процессов при несимметричных коротких замыканиях в нагрузке СГ. Несимметричные короткие замыкания задавались трехфазной (в осях $a, b, c)$ моделью статической нагрузки в форме (21).

При двухфазном коротком замыкании момент может в 1,3-1,4 раза превысить момент при трехфазном коротком замыкании из-за искажения синусоидальной формы момента и увеличения его пикового значения [21]. Как показало моделирование, еще более значительный момент возникает при отключении короткого замыкания и восстановлении связи генератора с ЭС.

Выводы. В статье на примере синхронного генератора рассмотрено математическое описание электрической машины в естественной трехфазной системе координат. Показано, что такие модели получены и апробированы уже довольно давно [20, 11], но до сих пор и во многом по традиции используется почти исключительно математическое моделирование в преобразованной двухфазной системе координат [7]. 
Это объясняется удобством алгоритмизации при компьютерном моделировании и увеличением наглядности за счет упрощений [7, 4]. Тем не менее, трехфазное рассмотрение и моделирование электрических машин и других элементов ЭС имеет и свои преимущества, особенно при исследованиях несимметричных режимов работы и несимметрии в электрических машинах.

Рассмотрено математическое представление такого описания в единой общей форме записи в векторно-матричном виде, подобно ранее рассматриваемому в [19] для описания $d, q$. Показана работоспособность такой формы математического описания при моделировании несимметричных режимов работы элементов электрических систем. Создание программных комплексов на базе этого описания предоставляет возможность моделировать структурно-сложные электрические системы при различных возмущениях [2]. Поэтому исследование этого вопроса представляется весьма актуальным. Тем более, что в последние годы отдельные авторы начинают проводить исследования в сходном направлении, вводя свои собственные унифицированные формы записи уравнений элементов [16] и во многом повторяя результаты, ранее полученные нами в [1] и [2].

В последующих работах авторы предполагают рассмотреть моделирование взаимодействия отдельных элементов ЭС (электрогенераторы, электродвигатели, элементы электрической нагрузки, линии электропередачи и др.) в рамках единой ЭС при представлении всех элементов в естественной трехфазной системе координат $a, b, c$, что дает возможность учитывать и исследовать несимметричные режимы в характерных точках произвольной конфигурации ЭС.

\section{Библиографический список}

1. Кавалеров Б.В. Математическое моделирование мини-энергосистем с газотурбинными установками ... дис. канд. техн. наук. Пермь: Изд-во Перм. гос. техн. ун-та, 2000. - 157 с.

2. Кавалеров Б.В. Методологические и теоретические основы автоматизации испытаний систем управления электроэнергетическими газотурбинными установками с учетом динамики электроэнергетической системы: дис. ... д-ра техн. наук: 05.13.06. - Пермь, 2012. - 409 с.

3. Бессонов Л.А. Теоретические основы электротехники. - М.: Высшая школа, 1996. - 640 с. 
4. Веников В.А. Переходные электромеханические процессы в электрических системах. - М.: Высшая школа, 1985. - 536 с.

5. Memon A.P. A simple simulation technique of proportional integral derivative excitation control of synchronous generator // Quarterly Mehran University research journal of engineering and technology. - 2002. - Vol. 21. № 1. - P. 39-44.

6. Винокур В.М., Кавалеров Б.В. Динамическая модель взаимодействия структурных элементов в системе электроснабжения // Информационные управляющие системы: сб. науч. тр. - Пермь: Изд-во Перм. гос. техн. ун-та, 2002. - С. 164-167.

7. Kundur P. Power system stability and control. - New York, McGraw-Hill, 1994. - 1176 p.

8. Веретенников Л.П. Исследование процессов в судовых электроэнергетических системах. Теория и методы. - Л.: Судостроение, 1975. - $376 \mathrm{c}$.

9. Сивокобыленко В.Ф. Математическое моделирование синхронной машины с многоконтурным ротором в фазных координатах // Технічна електродинаміка. - 2015. - № 1. - С. 51-58.

10. Славутский А.Л. Моделирование переходных режимов узла нагрузки с асинхронным двигателем в фазных координатах // Электротехнические комплексы и системы. - 2015. - № 1. - Т. 11. - С. 38-44.

11. Каган Б.М., Урман Е.Л. Расчет на цифровых вычислительных машинах переходных процессов в синхронных машинах по дифференциальным уравнениям с периодическими коэффициентами // Электричество. - 1961. - № 4. - С. 43-48.

12. Целемецкий В.А. Матричные математические модели электрических машин переменного тока в фазных координатах // Известия АН СССР. Энергетика и транспорт. - 1978. - № 2. - С. 113-122.

13. Гусейнов Ф.Г. Упрощение расчетных схем электрических систем. - М.: Энергия, 1978. - 184 с.

14. Жуков В.В. Короткие замыкания в узлах комплексной нагрузки электрических сетей. - М.: Изд-во МЭИ, 1994. - 224 с.

15. Жуков В.В. Короткие замыкания в электроустановках напряжением до 1 кВ. - М.: Изд-во МЭИ, 2004. - 192 с.

16. Ульянов С.А. Электромагнитные переходные процессы в электрических системах. - М.-Л.: Энергия, 1964. - 704 с. 
17. Kavalerov B. Algorithms of structural simulation for electric power supply systems / Acta Universitatis Pontica Euxinus // International Scientific Journal: Technical University of Varna, Ovidius University of Constanta, Perm State Technical University. - 2005. - Vol. 4, № 1. - P. 64-67.

18. Vinokur V., Kavalerov B. Mathematical simulation of autonomous mini-power stations // Proceedings of Second International Congress on Mechanical and Electrical Engineering and Marine Industry. - Varna, Bulgaria. 07-09 October 2005. - Vol. IV. - P. 112-115.

19. Кавалеров Б.В., Петроченков А.Б., Один К.А., Тарасов В.А. Моделирование взаимодействия структурных элементов электроэнергетических систем // Электротехника. - 2013. - № 1. - С. 11-15.

20. Веретенников Л.П., Целемецкий В.А. Общий алгоритм исследования несимметричных режимов в автономных энергетических системах // Электричество. - 1970. - № 6. - С. 20-26.

21. Беляев А.В. Защита, автоматика и управление на электростанциях малой энергетики. Ч. 1. - М.: НТФ «Энергопрогресс», 2010. - 84 с.

22. Radman G. Dynamic model for power systems with multiple FACTS controllers // Electric power systems research. - 2008. - Vol. 78. № 3. - P. 361-371.

23. Веприк Ю.Н., Небера О.А. Унифицированные модели элементов систем электроснабжения на основе уравнений в фазных координатах // Електротехніка і Електромеханіка. - 2015. - № 6. - С. 56-60.

24. Кетнер К.К., Козлова И.А., Сендюрев В.М. Алгоритмизация расчетов переходных процессов автономных электроэнергетических систем. - Рига: Зинатне, 1981. - 166 с.

\section{References}

1. Kavalerov B.V. Matematicheskoe modelirovanie mini-energosistem s gazoturbinnymi ustanovkami [Mathematical modeling of minipower systems with gas turbine plants]. Ph.D. thesis. Perm: Permskii gosudarstvennyi tekhnicheskii universitet, 2000. 157 p.

2. Kavalerov B.V. Metodologicheskie i teoreticheskie osnovy avtomatizatsii ispytanii sistem upravleniia elektroenergeticheskimi gazoturbinnymi ustanovkami s uchetom dinamiki elektroenergeticheskoi sistemy [Methodological and theoretical bases of automation of tests of control systems of electric power gas turbine installations taking into account the dynamics of the electric power system]. Ph.D. thesis. Perm, 2012. 409 p. 
3. Bessonov L.A. Teoreticheskie osnovy elektrotekhniki [Theoretical foundations of electrical engineering]. Moscow: Vysshaia shkola, 1996. 640 p.

4. Venikov V.A. Perekhodnye elektromekhanicheskie protsessy $\mathrm{v}$ elektricheskikh sistemakh [Transient Electromechanical processes in electrical systems]. Moscow: Vysshaia shkola, 1985. 536 p.

5. Memon A.P. A simple simulation technique of proportional integral derivative excitation control of synchronous generator. Quarterly Mehran University research journal of engineering and technology, 2002, vol. 21, no. 1, pp. 39-44.

6. Vinokur V.M., Kavalerov B.V. Dinamicheskaia model' vzaimodeistviia strukturnykh elementov $\mathrm{v}$ sisteme elektrosnabzheniia [Dynamic model of interaction of structural elements in the power supply system]. Informatsionnye upravliaiushchie sistemy: sbornik nauchnykh trudov. Perm: Permskii gosudarstvennyi tekhnicheskii universitet, 2002, pp. 164-167.

7. Kundur P. Power system stability and control. New York, McGraw-Hill, 1994. 1176 p.

8. Veretennikov L.P. Issledovanie protsessov $\mathrm{v}$ sudovykh elektroenergeticheskikh sistemakh. Teoriia i metody [Research of processes in ship electric power systems. Theory and methods]. Leningrad: Sudostroenie, 1975. $376 \mathrm{p}$.

9. Sivokobylenko V.F. Matematicheskoe modelirovanie sinkhronnoi mashiny $\mathrm{c}$ mnogokonturnym rotorom $\mathrm{v}$ faznykh koordinatakh [Mathematical modeling of synchronous machine with multi-circuit rotor in phase coordinates]. Tekhnichna elektrodinamika, 2015, no. 1, pp. 51-58.

10. Slavutskii A.L. Modelirovanie perekhodnykh rezhimov uzla nagruzki s asinkhronnym dvigatelem $\mathrm{v}$ faznykh koordinatakh [Simulation of transient modes of load node with asynchronous motor in phase coordinates]. Elektrotekhnicheskie kompleksy i sistemy, 2015, no. 1, vol. 11, pp. 38-44.

11. Kagan B.M., Urman E.L. Raschet na tsifrovykh vychislitel'nykh mashinakh perekhodnykh protsessov $\mathrm{v}$ sinkhronnykh mashinakh po differentsial'nym uravneniiam s periodicheskimi koeffitsientami [Calculation on digital computers of transients in synchronous machines by differential equations with periodic coefficients]. Elektrichestvo, 1961, no. 4, pp. 43-48.

12. Tselemetskii V.A. Matrichnye matematicheskie modeli elektricheskikh mashin peremennogo toka $\mathrm{v}$ faznykh koordinatakh [Matrix mathematical models of electric machines of alternating current in phase coordinates]. Izvestiia AN SSSR. Energetika i transport, 1978, no. 2, pp. 113-122. 
13. Guseinov F.G. Uproshchenie raschetnykh skhem elektricheskikh sistem [Simplification of design schemes of electrical systems]. Moscow: Energiia, 1978. $184 \mathrm{p}$.

14. Zhukov V.V. Korotkie zamykaniia v uzlakh kompleksnoi nagruzki elektricheskikh setei [Short circuits in the nodes of complex load of electrical networks]. Moscow: Moskovskii energeticheskii institut, 1994. 224 p.

15. Zhukov V.V. Korotkie zamykaniia v elektroustanovkakh napriazheniem do $1 \mathrm{kV}$ [Short circuits in electrical installations up to $1 \mathrm{kV}$ ]. Moscow: Moskovskii energeticheskii institut, 2004. 192 p.

16. Ul'ianov S.A. Elektromagnitnye perekhodnye protsessy $\mathrm{v}$ elektricheskikh sistemakh [Electromagnetic transients in electrical systems]. Moscow.-Leningrad: Energiia, 1964. 704 p.

17. Kavalerov B. Algorithms of structural simulation for electric power supply systems. Acta Universitatis Pontica Euxinus. International Scientific Journal: Technical University of Varna, Ovidius University of Constanta, Perm State Technical University, 2005, vol. 4, no. 1, pp. 64-67.

18. Vinokur V., Kavalerov B. Mathematical simulation of autonomous mini-power stations. Proceedings of Second International Congress on Mechanical and Electrical Engineering and Marine Industry. Varna, Bulgaria. 07-09 October 2005, vol. IV, pp. 112-115.

19. Kavalerov B.V., Petrochenkov A.B., Odin K.A., Tarasov V.A. Modelirovanie vzaimodeistviia strukturnykh elementov elektroenergeticheskikh sistem [Modeling of interaction of structural elements of electric power systems]. Elektrotekhnika, 2013, no. 1, pp. 11-15.

20. Veretennikov L.P., Tselemetskii V.A. Obshchii algoritm issledovaniia nesimmetrichnykh rezhimov $\mathrm{v}$ avtonomnykh energeticheskikh sistemakh [General algorithm of investigation of asymmetric regimes in Autonomous power systems]. Elektrichestvo, 1970, no. 6, pp. 20-26.

21. Beliaev A.V. Zashchita, avtomatika i upravlenie na elektrostantsiiakh maloi energetiki. Chast' 1 [Protection, automation and control of small power plants. Part 1]. Moscow: NTF "Energoprogress", 2010. 84 p.

22. Radman G. Dynamic model for power systems with multiple FACTS controllers. Electric power systems research, 2008, vol. 78, no. 3, pp. 361-371.

23. Veprik Iu.N., Nebera O.A. Unifitsirovannye modeli elementov sistem elektrosnabzheniia na osnove uravnenii v faznykh koordinatakh [Uni- 
fied models of elements of power supply systems based on equations in phase coordinates]. Elektrotekhnika i Elektromekhanika, 2015, no. 6, pp. 56-60.

24. Ketner K.K., Kozlova I.A., Sendiurev V.M. Algoritmizatsiia raschetov perekhodnykh protsessov avtonomnykh elektroenergeticheskikh sistem [Algorithmization of calculations of transients of Autonomous electric power systems]. Riga: Zinatne, 1981. 166 p.

\section{Сведения об авторах}

Кавалеров Борис Владимирович (Пермь, Россия) - доктор технических наук, доцент, заведующий кафедрой «Электротехника и электромеханика» Пермского национального исследовательского политехнического университета (614990, Пермь, Комсомольский пр., 29, e-mail:kbv@pstu.ru).

Кошелев Владислав Викторович (Пермь, Россия) - студент Пермского национального исследовательского политехнического университета (614990, Пермь, Комсомольский пр., 29, e-mail: the_vlad777@mail.ru).

\section{About the authors}

Kavalerov Boris Vladimirovich (Perm, Russian Federation) is a Doctor of Technical Sciences, Associate Professor, head of the department of electrical engineering and electromechanics Perm National Research Polytechnic University (614990, Perm, 29, Komsomolsky pr., e-mail: kbv@pstu.ru).

Koshelev Vladislav Viktorovich (Perm, Russian Federation) is a Student Perm National Research Polytechnic University (614990, Perm, 29, Komsomolsky pr., e-mail: the_vlad777@mail.ru).

Получено 17.07.2019 